\title{
Report from the 9th WASOG Meeting \& 11th BAL International Conference
}

\author{
O.P. Sharma
}

Monaldi Arch Chest Dis 2008; 69: 3, 89-90.

Correspondence: Om P. Sharma, FRCP Professor of Medicine, Keck School of Medicine, LAC+USC Medical Center, 1200 North State Street: Room 11-900,Los Angeles, CA 90033,USA; e-mail: osharma@usc.edu

The $9^{\text {th }}$ World Association of Sarcoidosis and Other Granulomatous Diseases (WASOG) Meeting, in association with Broncho-alveolar Lavage (BAL) International Conference, was held in Athens, Greece, June 19-22, 2008 (1). More than 250 international clinicians and scientists attended the conferences; they were 104 oral and poster presentations encompassing various clinical and basic science aspects of sarcoidosis and interstitial lung disease. From these outstanding presentations, we have selected a few that reflect interesting aspects and new information related to sarcoidosis. In the following Editorial, Dr. Reynolds will highlight the most important topics on BAL.

\section{Etiology}

The cause of sarcoidosis remains unknown. Dr. Lee Newman from Denver, USA argued that sarcoidosis should be considered a disease with many causes including bacteria, viruses, organic antigens, and inorganic dusts. Dr Wonder Drake from Nashville, Tennessee told us that the presence of mycobacterial virulence factors, ESAT-6 and $\mathrm{katG}$ antigens points toward a mycobaterial etiology. These antigens generate a CD4+ T cells immune response in the context of DRN1*1101 that plays a role the pathogenesis of sarcoidosis. Dr. Anna Dubaniewicz from Gdansk, Poland is also leaning towards a mycobacterial etiology of sarcoidosis. She put forward an interesting hypothesis that genetically different individuals manifest dissimilar immune responses to the same antigen. Thus, an undefined mycobacterium may produce two different syndromes, sarcoidosis or tuberculosis. Her studies on mycobacterial heat shock proteins are designed to explore relationship between sarcoidosis and tuberculosis. Dr. Osoinik and colleagues from Slovenia showed that invariant natural-killer T (iNKT) cells are deficient in bronchoalveolar fluid in sarcoidosis patients. It is, however, too early to say if it means that sarcoidosis has an autoimmune pathogenesis. There were many presentations that pointed out the similarities and coexistence of sarcoidosis with autoimmune disease and cancer. Dr. Oikonomidis from Ioannina, Greece showed that A549 cancer cell line showed significantly increased proliferation rate when treated with human sarcoidosis serum. The cell line when subjected to normal serum showed no cellular proliferation. The relationship between sarcoidosis and cancer, if any, remains enigmatic.

\section{Clinical Features}

Dr. Marjolein Drent from Maastricht, The Netherlands and Dr. Robert Baughman from Cincinnati, Ohio tackled the difficult problem of fatigue in sarcoidosis; Dr. Baughman emphasized that specific therapy may be useful in controlling symptoms of fatigue; whereas, Dr. Drent suggested that it would be more productive to treat the cause. Both speakers, however, agreed that fatigue is an important cause of disability in these patients and required more attention and understanding. Marjon Elfferich and her colleagues from Maastricht pointed out that the patients with fatigue and sarcoidosis have cognitive inefficiency that usually is not recognized. Dr. Mark Judson from Charleston, South Carolina explored the mystery of three difficult problems of upper airway involvement, lupus pernio, and myocardial sarcoidosis. In his experience sarcoidosis of the heart required no treatment if the patient has no irregular heart rate, arrhythmia or electro-cardiographic abnormalities. Dr. Teruo Tachibana from Osaka, Japan described his experience with liver disease in the Japanese sarcoidosis patients. He found that peritoneoscopy and abdominal CT were helpful in establishing the diagnosis. The liver involvement responded nicely to treatment with corticosteroids. Dr. Mana and colleagues examineed 507 patients with sarcoidosis and identified $86(17 \%)$ patients with biopsy proved granulomatous lesions involving the skin. Maculo-papular and subcutaneous nodules were observed mainly in patients 
with stage I diseases; whereas, plaques and lupus pernio occurred in stages II and III. The type of skin lesions provide prognostic information about the systemic disease.

\section{Prognosis}

Dr. Johan Grunewald told us that the patients with Lofgren's syndrome who are HLA-DRB $1 * 03$ positive have much better prognosis then the patients with Lofgren's syndrome who are HLADRB $1 * 03$ negative. More than $90 \%$ of the patient with DRB $1 * 03$ spontaneously subside as opposed to only $50 \%$ of those with HLA-DRB $1 * 03$ negative genotype.

\section{Treatment}

Most of the speakers agreed that the best treatment for sarcoidosis was the judicious use of the available including prednisone, methotrexate, hydroxychloroquine, azathioprine, and infliximab.

\section{Reference}

1. http://www.wasogbal2008.com/

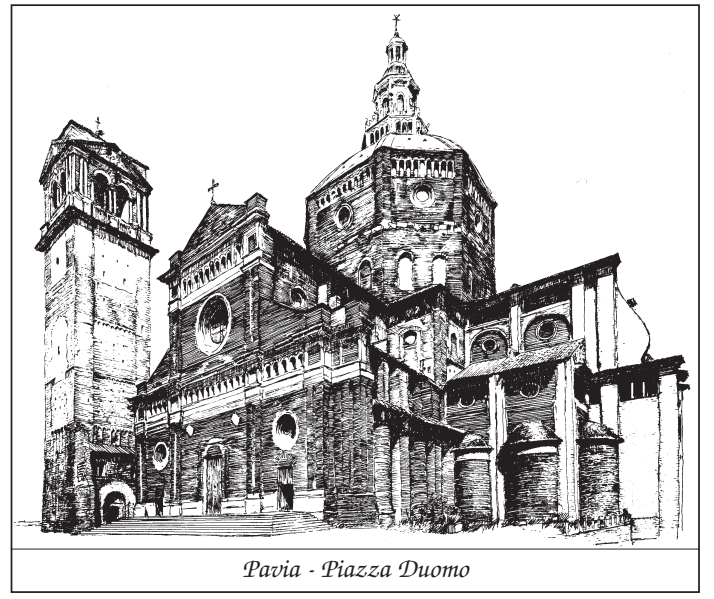

